\title{
Deciduoma, a Large Intrauterine Mass of Deciduosis
}

\author{
Megha Dasani, BA ${ }^{1}$ Hwa Jeong Lee, MD $^{2}$ Asha Rijhsinghani, MD ${ }^{1,3}$ \\ ${ }^{1}$ Department of Obstetrics and Gynecology, Albany Medical Center, \\ Albany, New York \\ 2 Department of Pathology, Albany Medical Center, Albany, New York \\ ${ }^{3}$ Division of Maternal and Fetal Medicine, Albany Medical Center, \\ Address for correspondence Asha Rijhsinghani, MD, Department of \\ Obstetrics and Gynecology, Division of Maternal and Fetal Medicine, \\ Albany Medical Center, 391 Myrtle Avenue, 2nd Floor, Albany, NY \\ 12208 (e-mail: rijhsia@mail.amc.edu).
}

Albany, New York

Am J Perinatol Rep 2019;9:e337-e340.

\begin{abstract}
Keywords

- intrauterine mass

- placenta

- deciduoma

- ectopic uterine tissue

Deciduosis is the presence of ectopic decidual tissue outside the uterus, pelvic, or abdominal organs usually associated with pregnancy. It usually presents as smaller lesions but can be larger vascular lesions. Typically, these masses are detected incidentally during operative procedures. Our patient was referred at 14 weeks for a large intrauterine mass detected on ultrasound examination that was initially thought to be an acardiac twin. The mass was highly vascularized. However, since the patient was asymptomatic, she strongly desired to continue the pregnancy. The pregnancy was followed closely from 14 to 39 weeks with serial ultrasound examinations. The vascularity was documented to diminish overtime and the mass appeared to convolute as well. Due to the decrease in vascularity of the mass, the patient was allowed spontaneous vaginal delivery at term. Following delivery of the fetus and the placenta, the mass was easily extracted manually without any complications.
\end{abstract}

Deciduosis is the presence of ectopic decidual tissue in locations outside the uterus. This is a phenomenon first described in 1887 by Walker. ${ }^{1}$ Deciduosis has been reported to occur in various pelvic and abdominal organs. ${ }^{2}$ Although international literature has only a few case reports published on ectopic deciduosis, the condition is more frequent than initially believed. Deciduosis is usually asymptomatic and can remain undetected throughout pregnancy. ${ }^{2}$ Based on a recent report, the condition was present in $10 \%$ of patients that underwent Cesarean section. ${ }^{2}$ Typically, deciduosis occurs during pregnancy but has been reported in nonpregnant women as well. In the absence of pregnancy, ectopic decidual changes are caused by progesterone released by the corpus luteum. ${ }^{3}$ During pregnancy, it is considered to be a benign lesion, not associated with any obstetric complications, and does not have any pathological impact during pregnancy. Total remission occurs in the postpartum period. ${ }^{2}$ Intrauterine deciduosis has not been previously reported. We described a case of a large vascular intrauterine mass initially suspected to be a malignant tumor, which regressed during the pregnancy, and was subsequently diagnosed as deciduosis.

received

May 4, 2019

accepted after revision

May 14, 2019
DOI https://doi.org/

10.1055/s-0039-1697647. ISSN 2157-6998.

\section{Case Report}

A 28-year-old female, G1 P0 was referred at $14^{4 / 7}$ weeks for reasons of abnormal twin gestation with early onset oligohydramnios. The referral document indicated a hydropic structure with cardiac activity and a normal second fetus with a heart rate of $145 \mathrm{bpm}$. The hydropic structure was identified as having prominent vessels and no extremities, and referred with diagnosis of suspected acardiac twin. The patient had no history of spotting, bleeding, discomfort, chronic headaches, or respiratory symptoms at the time of referral.

In our assessment the pregnancy was a singleton at $14^{4 / 7}$ weeks with a large vascular mass protruding into the lower uterine cavity, arising from the cervicouterine area (-Figs. 1 and 2). There was a small amount of fluid around the mass. The pulse rate in the blood vessels within the mass was similar to the maternal heart rate. The singleton fetus appeared normal with normal growth. The differential diagnosis included a pedunculated fibroid, a large vascular polyp, or a malignant growth. The patient was counseled regarding the possible causes and options, and that if the mass was

Copyright @ 2019 by Thieme Medical Publishers, Inc., 333 Seventh Avenue, New York, NY 10001, USA. Tel: +1(212) 584-4662.
License terms

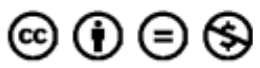




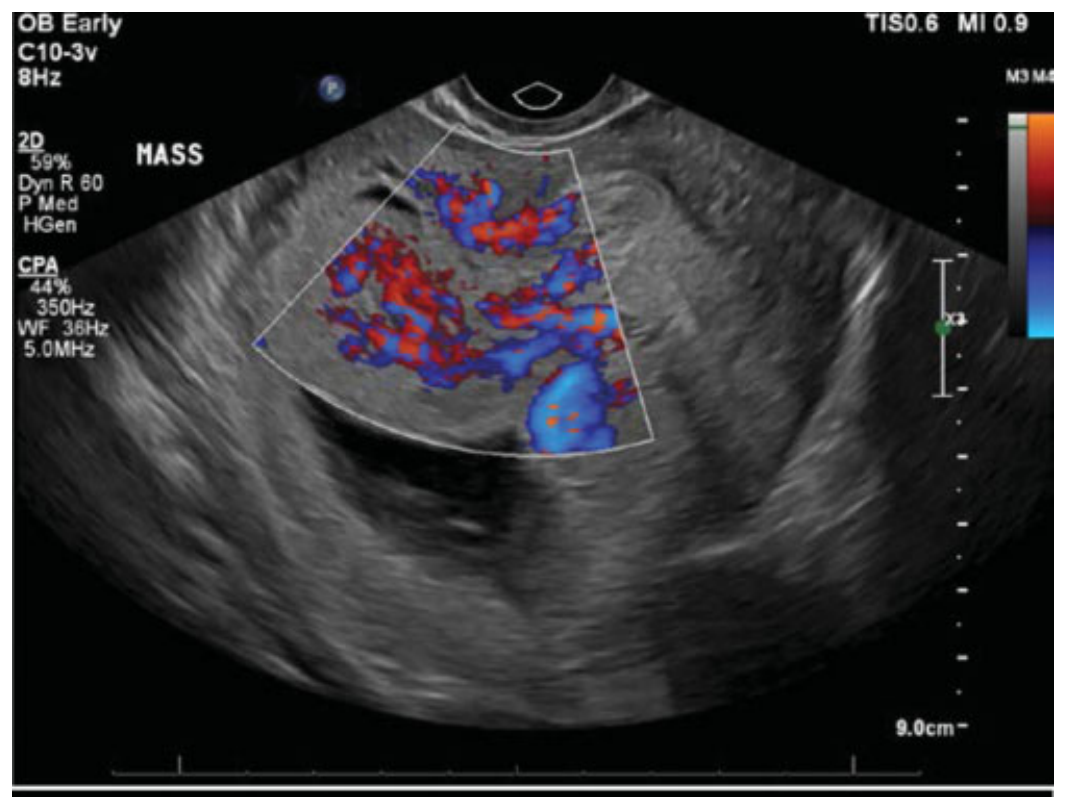

Fig. 1 Increased vascularity within the Intrauterine Mass at $14^{4 / 7}$ weeks' gestation.

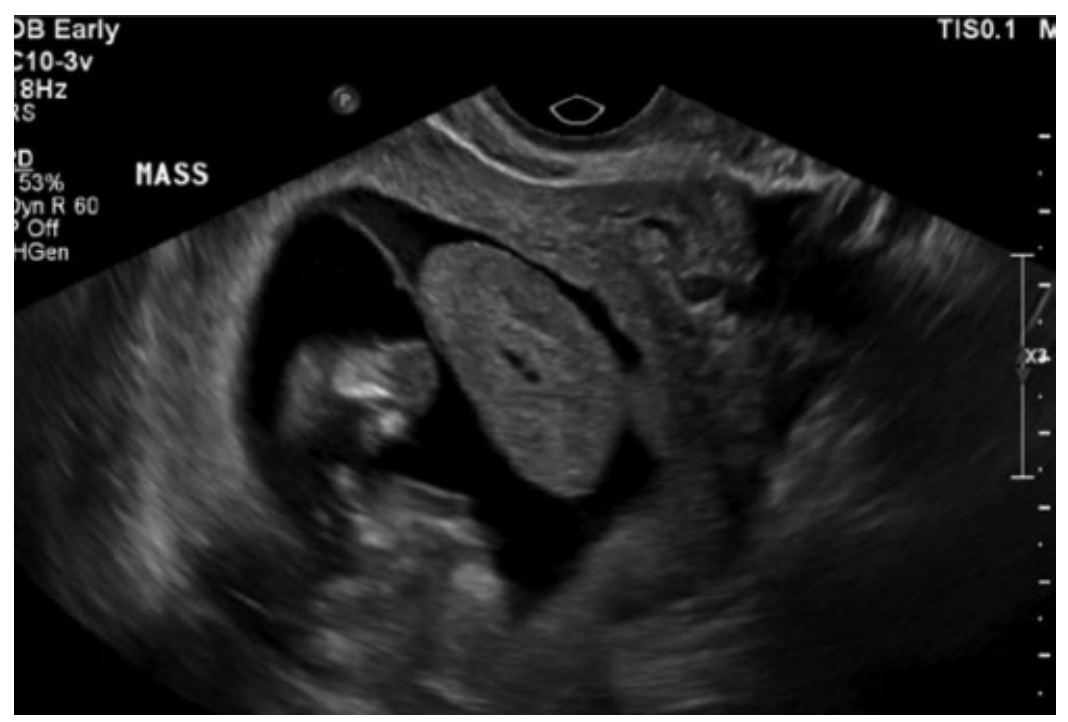

Fig. 2 Intrauterine mass surrounded by fluid, at $14^{4 / 7}$ weeks' gestation.

suspected to be malignant on further workup she would have the option of pregnancy termination. The patient declined to pregnancy termination irrespective of the diagnosis. A magnetic resonance imaging (MRI) was ordered, and the patient was subsequently referred for a consultation with the gynecologic services for assessment of the tumor.

An MRI at $18^{5 / 7}$ weeks reported a soft tissue mass of $1.6 \times 2.0 \times 4.9 \mathrm{~cm}$, eccentric and crescentic shaped with differential diagnosis of a "Stuck Twin syndrome" with demise of the presenting stuck twin. At $19^{3 / 7}$ weeks, on a repeat obstetrical ultrasound, the fluid surrounding the mass and the vascularity were significantly reduced. The mass continued to have minimal arterial circulation with a pulse rate consistent with the maternal heart rate.

Ultrasound examinations were initially repeated every month. At $21^{3 / 7}$ weeks, the mass stayed stable in size, measuring $4.36 \times 2.00 \times 3.26 \mathrm{~cm}$ and, at $25^{3 / 7}$ weeks, it was essentially unchanged measuring $4.7 \times 2.6 \times 1.8 \mathrm{~cm}$. Due to the stable size of the mass, as well as significantly reduced vascularity, the patient was reexamined at $33^{5 / 7}$ weeks and the mass had reduced in size measuring $3.6 \times 2.2$ $\times 3 \mathrm{~cm}$. Over time, the vascularity was documented to decrease with minimal blood flow. The mass stayed adherent to the lower portion of the posterior uterine wall.

At $39^{4 / 7}$ weeks, the patient experienced spontaneous labor and underwent a normal vaginal delivery. Following the delivery of the baby, the uterine cavity was explored and, with complete ease, the mass was manually extracted in full, with no additional blood loss. It was a smooth soft spongy mass with no raw edges (- Fig. 3). On pathology, the mass measured $7.5 \times 4.7 \times 2.6 \mathrm{~cm}$ and was described as an ovoid, membranous, dark red, soft tissue with a hemorrhagic surface. On the 


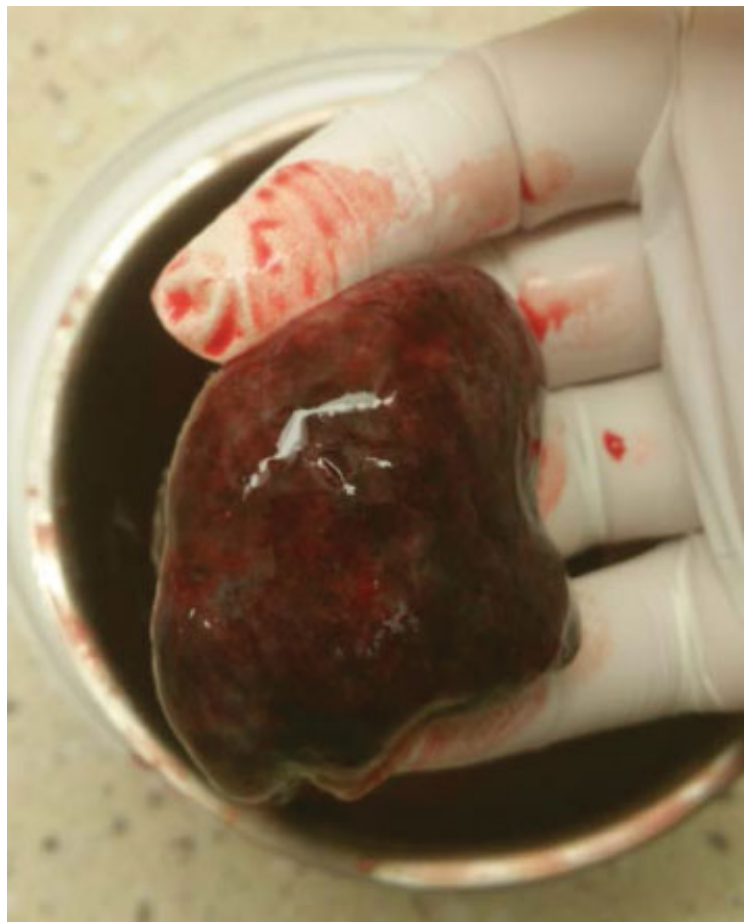

Fig. 3 Mass extracted during delivery, measured $7.5 \times 4.7 \times 2.6 \mathrm{~cm}$.

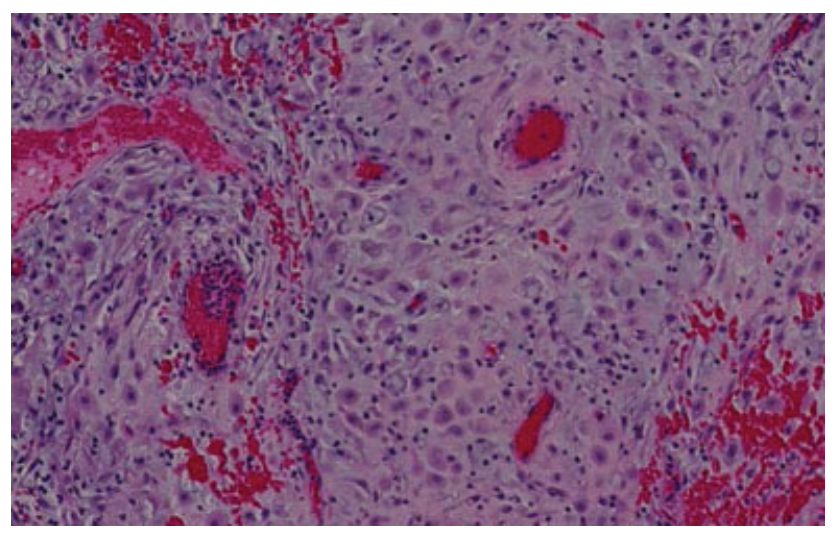

Fig. 4 Histopathology of the intrauterine mass.

final histopathologic diagnosis, the mass was reported as deciduosis (-Fig. 4). Three weeks after delivery a postpartum ultrasound was performed which was normal. Findings indicated anteverted normal appearing midline uterus and cervix. Endometrial stripe was normal. Ovaries were normal bilaterally with no cysts or masses. There was no evidence of the previously observed lower uterine mass.

\section{Discussion}

Ectopic deciduosis has been defined as decidual tissue found in an extrauterine location during a pregnancy. There are various theories on the pathogenesis of deciduosis. It has been suggested that progesterone can induce ectopic decidua, a reversible phenomenon. ${ }^{6,7}$ In association with pregnancy, the ectopic decidual cells have been reported in the fallopian tube, ovaries, uterine serosa, cervix, and vagina, as well as outside the genital tract including the peritoneum, omentum, appendix, and lymph nodes but not intrauterine. ${ }^{4,7}$ The lesions are described as white nodules less than $1 \mathrm{~cm}$ in diameter that may coalesce or be isolated. ${ }^{7}$ Ectopic decidua is usually an incidental finding, detected during surgical procedures, which include Cesarean sections, postpartum tubal ligations, and appendectomies. $^{7}$ Typically asymptomatic, deciduosis can present with clinical symptoms of shortness of breath, hemoptysis, pneumothorax, progressive anemia, pelvic pain, and risk of infections depending on the site. Rarely, complications, such as massive intra-abdominal bleeding have been reported. ${ }^{5}$

Though the lesions are typically described in $\mathrm{mm}$, larger lesions in the cervix and the omentum have been reported. The cervical lesions have been described as large masses with the largest reported being $8 \mathrm{~cm} .{ }^{4}$ In the omentum, the lesions can be large as well. When the mass is large, it is frequently mistaken for malignancy and requires histopathology for final diagnosis. ${ }^{2,4}$ Omental and peritoneal lesions are more common and with increasing duration of pregnancy regressive changes have been reported. ${ }^{8}$

To our knowledge, there are no reported cases of intrauterine deciduosis during the course of a normal pregnancy. In our case, we had the advantage of following the lesion longitudinally from the end of the first trimester until delivery at term. To our surprise, the vascularity, as well as the size of the mass, was seen to reduce remarkably by the third trimester, making it highly unlikely that we were dealing with a malignant tumor. At delivery we were prepared for an adherent mass and postpartum hemorrhage. However, the entire mass was easily extracted manually, without any complications. Fortunately, in our case, in spite of the lesion being large, the patient did not experience any episode of heavy bleeding antenatally or during the time of delivery. Perhaps, since the vascularity regressed overtime, the delivery was uncomplicated.

\section{Conclusion}

In conclusion, based on our case and those reported in the literature, we believe that there are two separate entities of ectopic deciduosis, diffuse deciduosis with smaller lesions and the larger vascular mass lesions. We propose to classify ectopic deciduosis into two categories, as benign diffuse ectopic deciduosis which is typically asymptomatic, and a deciduoma which is a large vascular lesion with potential for hemorrhage. By categorizing the larger vascular lesions as deciduoma, the lesion may be accurately diagnosed prenatally more frequently, and it could help the obstetrician prepare for the possible hemorrhagic complications. It would also guide the obstetrician in continuing with expectant management, with the hope of reduction in vascularity and size of the lesion as the pregnancy progresses.

Conflicts of interest

None.

\section{References}

1 Walker A. Der bau der Eihaeute bei Graviditatis abdominalis. Virch Arch Path Anat. 1887;197:72-99 
2 Markou GA, Goubin-Versini I, Carbunaru OM, Karatzios C, Muray JM, Fysekidis M. Macroscopic deciduosis in pregnancy is finally a common entity. Eur J Obstet Gynecol Reprod Biol 2016;197:54-58

3 Kinra P, Sen A, Sharma JC. Ectopic decidual reaction: a case report. Med J Armed Forces India 2006;62(03):280-281

4 Gornall AS, Naftalin NJ, Brown LJ, Konje JC. Massive necrosis of cervical ectopic decidua presenting in labour. BJOG 2000;107 (04):573-575

5 Lüdders DW, Henke RP, Saba M, Raddatz L, Soliman A, Malik E. Severe maternal pre- and postpartum intra-abdominal bleeding due to deciduosis. Geburtshilfe Frauenheilkd 2015;75(03):259-262
6 Ellis CL, Maleki Z, Ali SZ. Ectopic decidua in abdominal washings found intraoperatively at cesarean section. Diagn Cytopathol 2010; 38(10):740-741

7 Shukla S, Pujani M, Singh SK. Ectopic decidual reaction mimicking peritoneal tubercles: a report of three cases. Indian J Pathol Microbiol 2008;51(04):519-520

8 Büttner A, Bässler R, Theele C. Pregnancy-associated ectopic decidua (deciduosis) of the greater omentum. An analysis of 60 biopsies with cases of fibrosing deciduosis and leiomyomatosis peritonealis disseminata. Pathol Res Pract 1993;189(03): 352-359 